\title{
Spontaneous Isolated Dissection of the Posterior Inferior Cerebellar Artery Presenting with Lateral Medullary Infarction
}

\author{
Eun Hye Jeong, MD11, Hyun-Soon Jang, MD¹, Hyun-Jeung Yu, MD¹, Sook Young Roh, MD, PhD', \\ and Jong Won Choi, MD
}

Departments of ${ }^{1}$ Neurology and ${ }^{2}$ Neurosurgery, Bundang Jesaeng General Hospital, Seongnam, Korea

\begin{abstract}
Background: Spontaneous isolated dissection of the posterior inferior cerebellar artery (PICA) is rare and presents with variable clinical course and prognosis. We report a case of spontaneous isolated PICA dissection presenting with lateral medullary infarction.

Case Report: A 57-year-old female patient presented with right leg ataxia, headache, nausea and vomiting with a past medical history of hypertension. Neurological examination revealed right leg ataxia and left-side sensory change with right ptosis and miosis. The brain magnetic resonance imaging and magnetic resonance angiography showed an acute infarction in the right lateral medulla with abrupt luminal stenosis and subtle dilatation of the proximal segment of the right PICA. Transfemoral cerebral angiography showed an abrupt segmental stenosis of the right proximal PICA with a pre-stenotic fusiform dilatation, suggestive of dissection. The patient had a good clinical outcome with antiplatelet therapy.

Conclusion: We report a rare case of spontaneous isolated PICA dissection with lateral medullary infarction.

J Neurocrit Care 2014;7(2):137-140
\end{abstract}

Key Words: Posterior inferior cerebellar artery; Dissection; Infarction

\section{INTRODUCTION}

Spontaneous isolated dissection of the posterior inferior cerebellar artery (PICA) occurs rarely, because most PICA dissection occur secondarily to an extension of the vertebral artery dissection. ${ }^{1-4}$ The PICA is the largest branch of the vertebral artery and has many variations. Therefore, PICA dissection presents with variable clinical courses and prognosis. Clinical manifestations may be ischemic, hemorrhagic or both, depending on the dissecting plane. However, most of the reported cases presented with subarachnoid hemorrhage or intraventricular hemorrhage..$^{1-3}$ Here, we report a case of spontaneous isolated PICA dissection presenting with lateral medullary infarction.

Received: May 31, 2014 / Revised: July 12, 2014

Accepted: July 22, 2014

Address for correspondence: Eun Hye Jeong, MD Department of Neurology, Bundang Jesaeng General Hospital, 20 Seohyeon-ro 180beon-gil, Bundang-gu, Seongnam 463-774, Korea Tel: +82-31-779-0879, Fax: +82-31-779-0897

E-mail: gracej3@naver.com

\section{CASE REPORT}

A 57-year-old female patient presented with right leg ataxia, starting 10 hours ago. She also complained of dull headache in the right parieto-temporal area combined with nausea and vomiting, which was started 14-15 hours ago. There were no other symptoms such as weakness, sensory change or diplopia. She had a past medical history of hypertension on medication for 2-3 years. She had no previous history of diabetes mellitus, cerebrovascular attack, heart disease or trauma. On initial neurological examination, there was no motor weakness, but decreased sensations of pain and temperature were observed on the left extremities. Ataxia in the right leg and slightly hyperactive right knee jerk were also observed. One day after admission, right ptosis and miosis were developed without ipsilateral anhidrosis. There were no diplopia, gaze palsy, nystagmus and dysarthria.

Routine blood tests were unremarkable. The brain magnetic resonance imaging (MRI) was performed 2 days after the onset of symptoms and showed an acute infarction in 

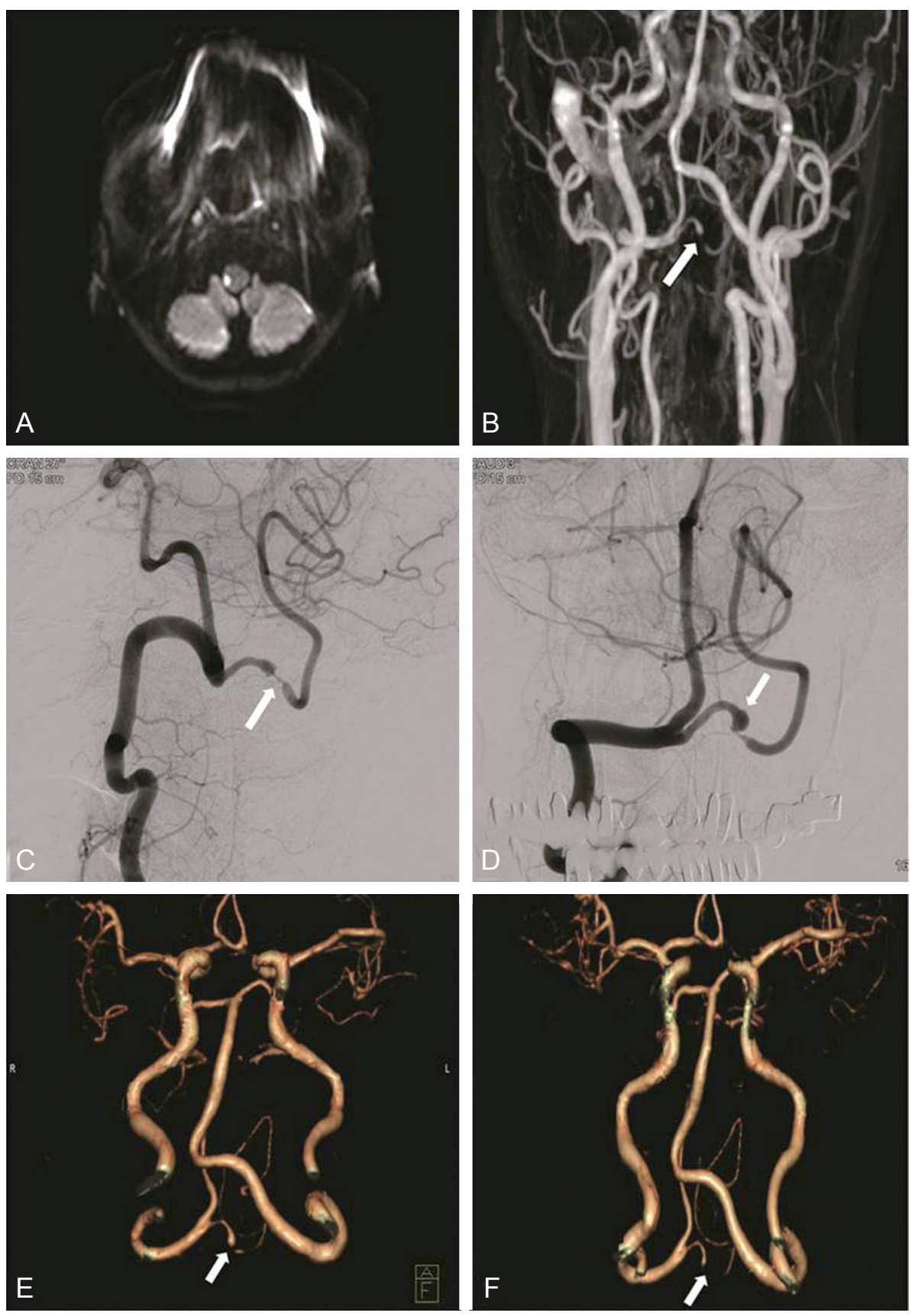

Figure 1. Brain MRI was performed 2 days after the onset of symptoms. Diffusion-weighted images revealed an acute infarction in the right lateral medulla (A). MRA showed an abrupt luminal stenosis (arrow) and subtle dilatation of the proximal segment of the right posterior inferior cerebellar artery (PICA), suggestive of possible dissection (B). Initial transfemoral cerebral angiography (TFCA), performed 4 days after the onset of symptoms showed an abrupt segmental stenosis (arrow) of the right proximal PICA $(C)$. There was an additional finding of a prestenotic fusiform dilatation (arrow) of the right proximal PICA on the follow-up TFCA 1 week later (D). Serial brain CT angiographies showed no changes of the stenosis with prestenotic fusiform dilatation (arrows) 4 months (E) and 10 months $(F)$ after the onset of symptoms.

the right lateral medulla (Fig. 1A). The magnetic resonance angiography (MRA) showed an abrupt luminal stenosis and subtle dilatation of the proximal segment of the right PICA, suggestive of possible dissection (Fig. 1B). The transfemoral cerebral angiography (TFCA), performed 4 days after the onset of symptoms showed an abrupt segmental stenosis of the right proximal PICA, which is suggestive of dissection (Fig. 1C). The origin of PICA was extracranial and dissection occurred at the lateral medul- lary segment of PICA, which was intradural. The followup TFCA 1 week later showed no change of stenosis of the right proximal PICA, but an additional finding of a pre-stenotic fusiform dilatation ( $3 \times 4 \mathrm{~mm})$ of the right proximal PICA was newly observed (Fig. 1D). Since 10 days had passed after dissection, a procedure-related risk from stent insertion or stent-assisted coil embolization was thought to be larger than a rupture risk. Therefore, we decided on a conservative management with antiplatelet therapy and 
planned for a follow- up TFCA 2 weeks later.

The patient's headache subsided 10 days later and the ataxia of the right leg improved enough that she was able to walk by herself. The patient was discharged with mild right ptosis and left leg sensory change. There were no changes on the follow-up TFCA 4 weeks after the onset of symptoms. There were also no changes on the brain computed tomography angiography (CTA) 4 months and 10 months after onset (Fig. 1E, 1F) and nearly all neurologic deficits of the patient were disappeared. So, the patient was planned to have a regular outpatient-based follow-up with antiplatelet therapy 6 months later.

\section{DISCUSSION}

There have been few case reports on isolated PICA dissection. One reason may be that the clinical presentations of vertebral artery dissection and isolated PICA dissection are not distinguishable. Also, because of its small diameter and tortuous course, it may be difficult to detect subtle abnormalities of PICA by a conventional MRI or MRA. Moreover, because cerebral angiography is generally not considered in cases with infarction unlike in cases of hemorrhage, the diagnosis of PICA dissection with ischemia can be underestimated.

The causes for isolated PICA dissection are not known for sure, but several conditions associated with dissection such as hypertension, trauma, excessive physical activities or specific arteriopathy have been suggested to be predisposing factors. ${ }^{2}$ Our case was thought to have a spontaneous dissection since there were no past histories of trauma or neck manipulation. PICA dissection can present as either infarction or hemorrhage, or as both. The proximal PICA dissection tends to present with infarction, while the distal portion tends to present as hemorrhage. ${ }^{5-7}$ Infarction due to PICA dissection may occur as lateral medullary syndrome or cerebellar infarction. Isolated PICA dissection accounts for only $2.9 \%$ of the lateral medullary infarction. ${ }^{8}$ Our patient presented with typical symptoms of lateral medullary syndrome, such as ipsilateral ptosis, miosis and ataxia with contralateral hemibody sensory change.

Definite diagnosis of PICA dissection is usually made by cerebral angiography. Dissection of the PICA may be found on cerebral angiography with normal MRA findings. Cerebral angiography usually shows segmental stenosis with fusiform or saccular dilatation of the artery (pearl and string sign), tapered narrowing, dissecting aneurysm and rarely ‘double lumen' findings. ${ }^{2,3}$ Finding of stenosis with fusiform or saccular dilatation is dominant in PICA dissection with hemorrhage, while stenosis without dilatation is dominant in PICA dissection with ischemia. ${ }^{9}$ According to a recent study, susceptibility-weighted imaging may be more helpful to detect the abnormality of PICA than conventional MRI or MRA. ${ }^{10}$

Treatment of PICA dissection is based on the manifestations of the infarction or hemorrhage. In general, conservative management with medical therapy such as anticoagulation or antiplatelet therapy is considered for ischemic patients, but it is still controversial in the aspects of safety or efficacy. ${ }^{2,3}$ Especially in patients with intracranial dissection, it may be problematic with anticoagulation or antiplatelet therapy, because of the possibility of extension of the intramural hematoma or subarachnoid hemorrhage. Some authors reported that anticoagulation or antiplatelet therapy may be relatively safe in patients with intracranial dissection. ${ }^{11}$ However, in patients with hemorrhage, early surgical or endovascular procedures may be needed to avoid additional bleeding. The treatment plan has to be modified individually considering the location, severity and progression of the dissection. ${ }^{2}$

In our case, although there was a fusiform dilatation on the follow-up TFCA, we did not choose endovascular or surgical treatment. We treated the patient with antiplatelet agent along with the short-term follow-up TFCA and brain CTA. The patient showed a good clinical outcome as well as a stable course.

\section{REFERENCES}

1. Tikkakoski T, Leinonen S, Siniluoto T, Koivukangas J. Isolated dissecting aneurysm of the left posterior inferior cerebellar artery: endovascular treatment with a Guglielmi detachable coil. AJNR Am J Neuroradiol 1997;18:936-8.

2. Tawk RG, Bendok BR, Qureshi AI, Getch CC, Srinivasan $\mathrm{J}$, Alberts $\mathrm{M}$, et al. Isolated dissections and dissecting aneurysms of the posterior inferior cerebellar artery: topic and literature review. Neurosurg Rev 2003;26:180-7.

3. Sedat J, Chau Y, Mahagne MH, Bourg V, Lonjon M, Paquis 
P. Dissection of the posteroinferior cerebellar artery: clinical characteristics and long-term follow-up in five cases. Cerebrovasc Dis 2007;24:183-90.

4. Shin YS, Kim HS, Kim SY. Stenting for vertebrobasilar dissection: a possible treatment option for nonhemorrhagic vertebrobasilar dissection. Neuroradiology 2007;49:149-56.

5. Kanou Y, Arita K, Kurisu K, Ikawa F, Eguchi K, Monden S, et al. Dissecting aneurysm of the peripheral posterior inferior cerebellar artery. Acta Neurochir (Wien) 2000;142:1151-6.

6. Yamakawa H, Kaku Y, Yoshimura S, Ohkuma A, Sakai N. Two cases of dissecting aneurysm of the distal posterior inferior cerebellar artery: possible involvement of segmental mediolytic arteriopathy in the pathogenesis. Clin Neurol Neurosurg 2005;107:117-22.

7. Ramgren B, Cronqvist M, Romner B, Brandt L, Holtås S, Larsson EM. Vertebrobasilar dissection with subarachnoid hemorrhage: a retrospective study of 29 patients. Neuroradiology 2005;47:97-104.

8. Kim JS, Lee JH, Choi CG. Patterns of lateral medullary infarction: vascular lesion-magnetic resonance imaging correlation of 34 cases. Stroke 1998;29:645-52.

9. Sasaki O, Ogawa H, Koike T, Koizumi T, Tanaka R. A clinicopathological study of dissecting aneurysms of the intracranial vertebral artery. Neurosurgery 1991;75:874-82.

10. Park MG, Choi JH, Yang TI, Oh SJ, Baik SK, Park KP. Spontaneous isolated posterior inferior cerebellar artery dissection: rare but underdiagnosed cause of ischemic stroke. J Stroke Cerebrovasc Dis 2014;23:1865-70.

11. Li S, Yan B, Kaye A, Mitchell P, Dowling R, Collins M, et al. Prognosis of intracranial dissection relates to site and presenting features. J Clin Neurosci 2011;18:789-93. 